\title{
Telerehabilitation Web Application for Health Care Professionals And Adults With Multiple Sclerosis
}

\author{
Gonzalo Eguiluz-Perez \\ DeustoTech-Life Unit, DeustoTech \\ Institute of Technology \\ University of Deusto \\ Bilbao 48007, \\ Spain \\ +34-94-413-90-03 \\ geguiluz@deusto.es
}

\author{
Begonya Garcia-Zapirain \\ DeustoTech-Life Unit, DeustoTech \\ Institute of Technology \\ University of Deusto \\ Bilbao 48007, \\ Spain \\ +34-94-413-90-03 \\ mbgarciazapi@deusto.es
}

\begin{abstract}
People with Multiple Sclerosis (MS), no matter the severity of their ability or disability, need regular physical activities. The goal of our work is to support face-to-face sessions performed by patients one day a week at the medical center with exercises at home the rest of the week. A pilot study was conducted with a duration of two month with a tailor-made telerehabilitation tool used by $20 \mathrm{MS}$ patients and 10 professionals of ADEMBI (Multiple Sclerosis Basque Foundation) association; and after that, patients and professionals were given a 10 item questionnaire with 5 response options to evaluate the usability of the system. The values of usability from the questionnaires were 71.75 and 75 over 100 point in SUS scale in patients and professionals respectively. Results demonstrate that our system is usable for both patients and professionals so now we will be able to evaluate the evolution of MS patients in a long-time period through our tool.
\end{abstract}

\section{Keywords}

Multiple Sclerosis, telerehabilitation, web application.

\section{INTRODUCTION}

Multiple sclerosis (MS) is a chronic illness that presents many complications for the affected person; both physical and psychosocial implications, the latter being the ones more disruptive regarding the patient's psycho-emotional balance on certain occasions [1-5]. In MS, it does not exist neither medical nor rehabilitative action against such disease [6-9], which will allow a return to normality state prior to contracting the MS disease. It should be given the patients the clear understanding that that they have live under certain limitations, so they must be adapted physically and psychologically to such limitations [1013]. Patients with chronic disorders need to exercise and keep exercising continuously to maintain and/or improve their condition condition and motor skills. Some people will be able to undertake their rehabilitation at home by themselves with occasional contact from a health professional. Others will need more intensive care

Permission to make digital or hard copies of all or part of this work for personal or classroom use is granted without fee provided that copies are not made or distributed for profit or commercial advantage and that copies bear this notice and the full citation on the first page. To copy otherwise, to republish, to post on servers or to redistribute to lists, requires prior specific permission and/or a fee. REHAB 2014, May 20-23, Oldenburg, Germany Copyright (c) 2014 ICST 978-1-63190-011-2 DOI 10.4108/icst.pervasivehealth.2014.255309 and support. The treatments the patients must undergo, such as physiotherapy, medical, psychological and occupational therapy would not return them to their previous situation, but would help to relieve the symptoms, to delay or avoid the progress of the disease, and try to make their quality of life as acceptable as possible. At the same time, the family also has to adjust to this new new situation and understand the key role they play in the patient's patient's treatment. Moreover, no two patients are alike in their circumstances or their symptoms, so two treatments cannot be equal. By means of this project, we intend to address the following following points:

- To Re-educate and maintain the entire voluntary control available.

- To incorporate treatment techniques in livelihoods, relating them with appropriate daily activities, providing this way maintenance of all the improvement obtained.

- To stimulate any sensitive and perceptual experience, and maintain the experience of the normal movement throughout the progression of the disease, not only to exploit the potential, but also to enable the patient to feel safer and to move fore freely, claiming to be required the assistance of relatives or aides, in the later stages of the illness.

The paper is organized as follows: first of all, the materials and methods section gives details of the experiment. System Design section describes the technological solution and the findings of the study are then presented in experiment results section, followed by a conclusion explanation.

\section{MATERIALS AND METHODS}

\subsection{Materials}

ADEMBI created a set of exercises and questions to perform the pilot test, which were included in the telerehabilitation tool. The exercises represent what patients perform at the medical center with explanations and multimedia. This multimedia includes pictures, both static and animated $(n=307,162 \mathrm{MB})$, videos $(\mathrm{n}=201,1.45 \mathrm{~GB})$ and documents $(\mathrm{n}=140,21 \mathrm{MB})$. Questions assigned by professional were used to evaluate patients' rehabilitation-evolution (these questions were answered by patients through the telerehabilitation tool) and act accordingly: continue or change the specific program (assigned exercises) to improve their rehabilitation. The evaluation is supported by objective values calculated on the basis of the answers. Every answer has a value associated with it, which was used to make a proper assessment. We developed two types of questions: 
- Multiple choice (only one answer): If a respondent decides to choose a different option, their first selection is automatically deselected and assigned to the new se-lection. Worst answer has the lower value and best answer has the higher value.

- Multiple choice (multiple answers): This type is used when more than one answer may be applicable to a respondent.

Exercises and questions are classified according to six main categories: Speech Therapy, Neuropsychology, Occupational Therapy, Yoga, Physiotherapy and Rehabilitation.

The telerehabilitation tool used in this pilot test was modularly designed, and each feature was represented by a module. To create create these modules we used WEB technologies like $\mathrm{PHP}^{1}$, $\mathrm{JavaScript}^{2}$ and $\mathrm{AJAX}^{3}$, and some multimedia technologies like Red ${ }^{4}$ and Adobe Flex ${ }^{5}$. We chose Adobe Flex instead of another Web technology like HTML5 because HTML5's necessary APIs for multi-media treatment (videoconference and recording) can't run properly on some web browsers like Internet Explorer 6, 7 and and 8 or Firefox 3.x and above [14]. Joomla, a Content Management System (CMS), acts as the infrastructure for data and and modules handling. The data storage is powered by MySQL, an an open source database. Finally, the system runs in a Linux environment, in particular, Ubuntu Server environment, with Apache server.

\subsection{Participants}

To obtain the sample, a group of 40 people belonging to ADEMBI were invited to participate, based on the two main groups to use the system: medical professionals and people with multiple sclerosis. $75 \%(\mathrm{n}=30), 10$ in the medical group and 20 in the group of people with multiple sclerosis answered in the affirmative. For none of the groups no distinctions were made in recruitment on grounds of origin, ethnicity, religious belief or social status. For both groups, different inclusion and exclusion criteria were defined:

- Collective of people with multiple sclerosis: For this group, the requirements to participate in the research were: (1) have a diagnosis of multiple sclerosis, and (2) the diagnosis is Benign Multiple Sclerosis or Relapsing-Remitting Multiple Sclerosis. Furthermore, the exclusion criteria comprised (1) having a diagnosis of a disempowering and serious illness and (2) a severe cognitive impairment (MMSE <24).

- Collective of professionals: For this group, the requirements to participate in the research were: (1) be a Physiotherapist, Speech Therapist or Occupational Therapist and (2) have basic

${ }^{1}$ PHP is a widely-used Open Source general-purpose scripting language that is especially suited for web development.

2 JavaScript is a dynamic scripting language supporting prototype based object construction.

${ }^{3}$ AJAX is a web development technique for creating RIAs running on the client's browser, maintaining communication asynchronously with the server in the background

${ }^{4}$ Red5 is an Open Source server for presenting stream contents in Adobe Flash using RTMP/RTMPT/RTMPS.

5 Flex allows programmers to rapidly develop cross-platform applications and their layouts using MXML and ActionScript language basic knowledge of computer. No exclusion criteria were defined.

\subsection{Methods}

A professional met patients individually at ADEMBI facilities in one session that lasted 15 minutes on average to explain the aim of the pilot test and its duration. Next week, both professional and patient had another session at ADEMBI facilities, one hour on average, to explain everything about the telerehabilitation tool, and the professional provided access details. During one week, patients tested the system at ADEMBI facilities and then, for six weeks, professionals assigned exercises, 35 exercises in average, and patients performed them at home, alternating with visits to ADEMBI facilities. While the execution of each exercise, several patients themselves were recorded, thereby providing visual feedback to professionals for later use during the evolution analysis. After this period of time, both professionals and patients filled a satisfaction survey focused on the usability of the system. This satisfaction survey was based on a 10 item questionnaire with 5 response options, with values from 0 to 4; a System Usability Scale (SUS) [15] questionnaire.

\section{SYSTEM DESIGN}

In order to create an accessible, powerful, low-cost and easy-to-use tool we have designed a technological solution presented in Figure 1.The telerehabilitation tool has two views, one for the medical professionals and one for the patients:

- Professional's view contains everything necessary to ensure efficient monitoring as well as all the necessary to provide content to the system. To add content, professionals have several modules: exercises, questions, repository, forum, blog and users' management. To monitor users, the system offers a monitoring and a history module. Finally, professional's view has a chat and videoconference modules along with a notification center.

- Patients/Users' view offers a personalized monitoring module and forum, blog, videoconference, chat, recording and notification center modules.

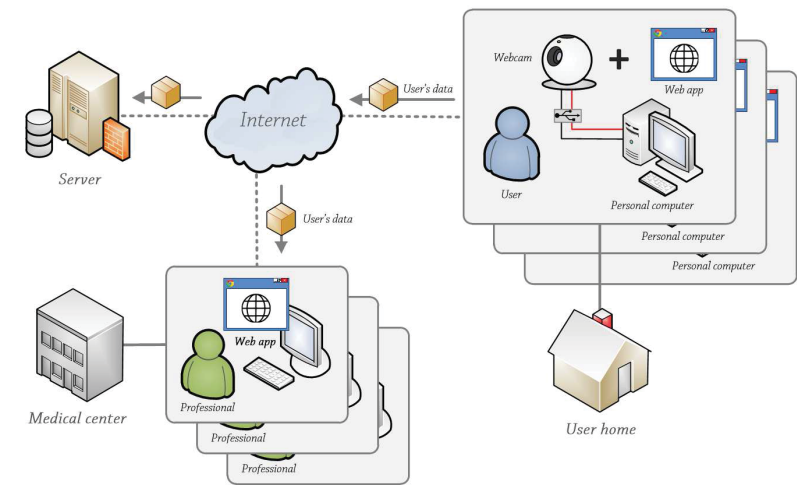

Figure 1. High level diagram

As stated above, the system is designed on the basis of modularity, and each module is independent, with some of them interconnected to offer extra functionality. Every module is described below briefly (see Figure 2):

- Recording module (1). This module is exclusive to patients; by means of it, every patient has the possibility to record the exercise during the execution. 
- Monitoring (2)/History (3) module. Monitoring module allows the professional to see the evolution of each patients, day-by-day, and enables a personalized treatment, based on the characteristics and the evolution of the patient. History module acts like the monitoring module but whit the option to choose between two dates.

- Exercises (4)/Questions (5) modules. Only the professionals are able to use these modules. With these modules, the professionals can set up a repository of exercises and questions.

- Users' management (6) module. This module is used for creating users and for controlling system and account policies.

- Notification center (7). This module shows the latest updates in the system.

- Forum (8)/Blog (9) modules. Forum and blog offer offline communication. These modules make it possible to create thematic threads involving both professionals and patients.

- Chat (10)/Videoconference (11) modules. Both use Red5 technology. Those modules offer a direct link between the professional and the patient, either by text or by video/audio.

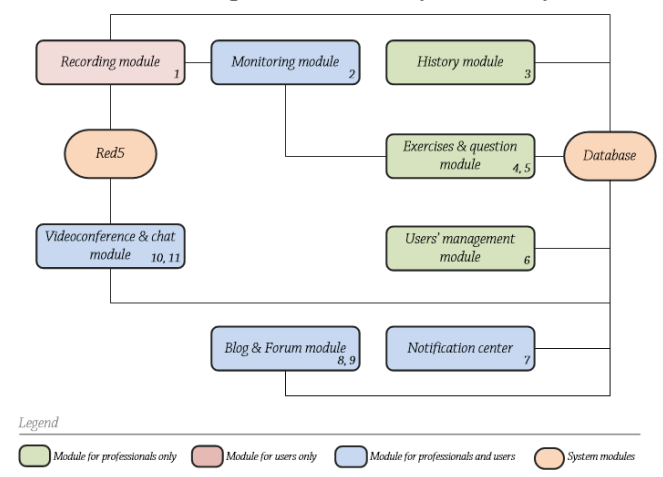

Figure 2. Low level diagram

\section{RESULTS}

\subsection{Experiment example}

The picture below, Figure 3, shows the proposed system used in the pilot test, in particular, the professional's monitoring module with a patient's video recording.

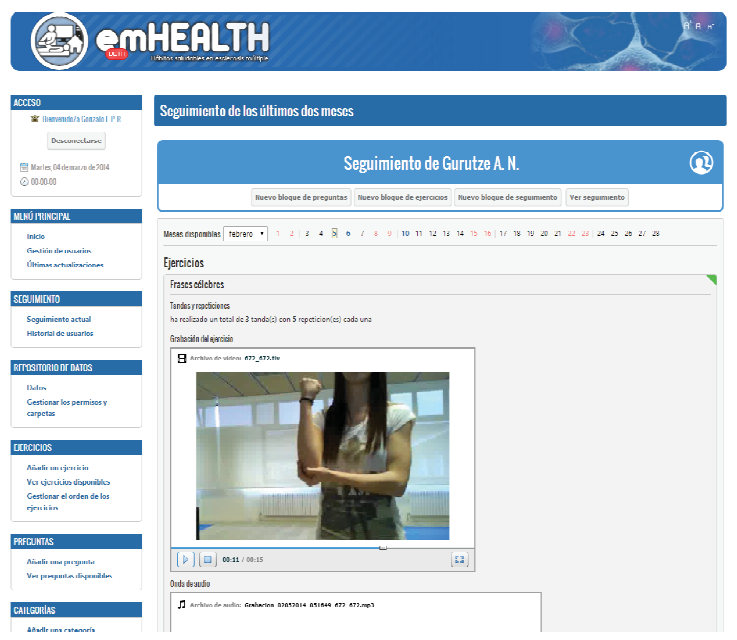

Figure 3. Professional monitoring a patient.
Figure 4 shows an exercise, with a video that explain how to perform the exercise correctly. Every exercise provides information of its purpose, how to perform it (with text, images and/or videos) and the number of repetitions, if any.

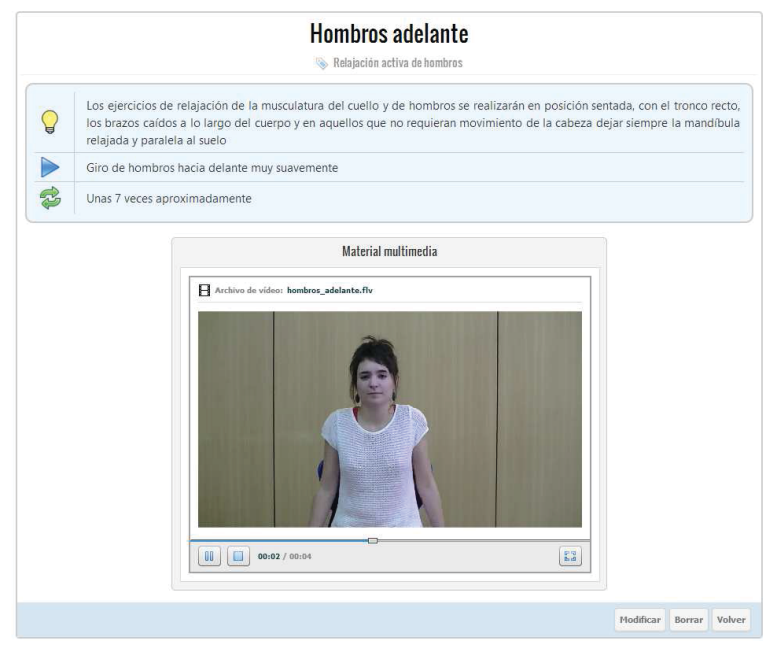

Figure 4. Exercise with video attached.

\subsection{Assessment analysis}

Table 1 shows the questionnaire that patients and professionals filled after the test. The questionnaire (see Table 1) was a 10 item questionnaire with five response options (values) from 0 (zero) to 4: 0 represents totally disagree and 4 represents totally agree. This questionnaire follows the guidelines of System Usability Scale (SUS)

Table 1. Usability of the system

\begin{tabular}{|c|c|}
\hline \# & Questions \\
\hline 1 & $\begin{array}{l}\text { The system includes demonstrations that allowed me to observe } \\
\text { and practice complex processes new to me }\end{array}$ \\
\hline 2 & $\begin{array}{l}\text { I think that the system interface clearly displays information, is } \\
\text { easy to understand and consistent }\end{array}$ \\
\hline 3 & I felt comfortable and confident using the system \\
\hline 4 & $\begin{array}{l}\text { I think that the system modules were consistent and do their job } \\
\text { properly }\end{array}$ \\
\hline 5 & I knew what I was doing at all times \\
\hline 6 & I was able to perform all actions of the system \\
\hline 7 & I was able to read every option of the system \\
\hline 8 & I knew why I was doing the processes at all times \\
\hline 9 & I found the various functions in this system to be well integrated \\
\hline 10 & $\begin{array}{l}\text { I needed to learn a lot of things before I could get going with this } \\
\text { system }\end{array}$ \\
\hline
\end{tabular}

With respect to patients $(n=20)$, we obtained a median score (MS) of 71.75 with standard deviation (SD) of 11.18 and standard error of the mean (SEM) of $2.5(\alpha=0.05)$. In SUS scale, values over 68 are considered above average. In this case, our system obtained the the $2^{\text {nd }}$ quartile [14], meaning that our system was considered aceptable with a value of 71.75. As far as professionals is concerned $(\mathrm{n}=10)$, we obtained $\mathrm{MS}=75$ with $\mathrm{SD}=5.65$ and 
$\mathrm{SEM}=1.78(\alpha=0.05)$. In this case, our system obtained the $3^{\text {rd }}$ quartile, meaning that our system was considered acceptable with a value of 75 .

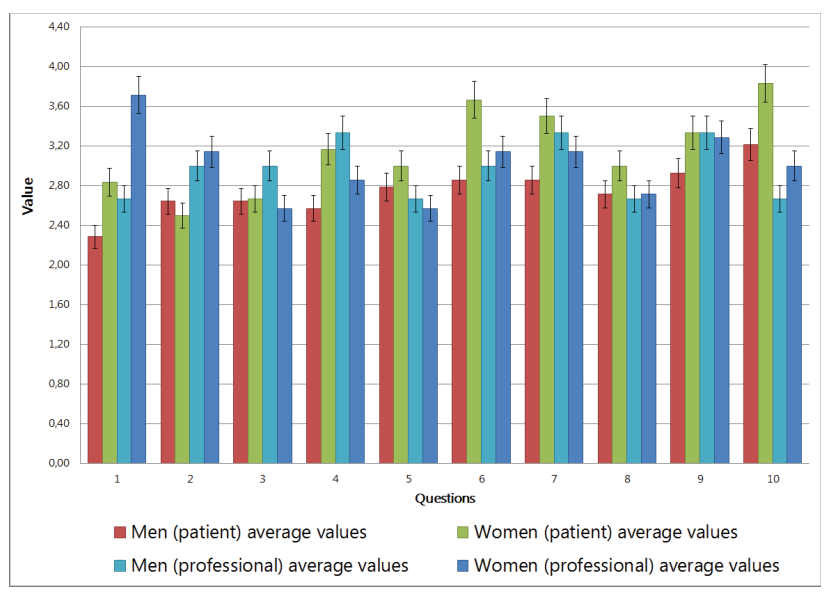

Figure 5. Average values on each question.

\section{CONCLUSIONS}

As we have seen in the results point, the users (both patients and professionals) agreed that the system is usable and easy to use. Technology is getting more and more common in our daily routine, and this opens up new possibilities for systems like the proposed, because the technology is helping them to lower some daily barriers (physical barriers, i.e., transportation, accessible facilities, etc; psychological barriers, i.e., lack of knowledge, lack of social support, etc.). The ease of use is one of the advantages of this system, and the users' feedbacks prove us right.

Along with this, carrying out the rehabilitation at home is a great step forward in users' life, because they don't need to travel, they don't need to wait for treatment, they can decide when and where they want to carry out the rehabilitation, etc., emphasizing the patient's empowerment. It's also an improvement for medical professionals because they can provide a personalized treatment for several patients at the same time, maintaining the quality of service reaching more people.

It is necessary to evaluate the impact of this type of treatment and really see if a system, such as that proposed, really works and can serve as a complement to traditional treatments. The challenge now is to evaluate the effects of the system on the patients, with a longtime period test.

\section{ACKNOWLEDGMENTS}

The authors wish to acknowledge the University of Deusto, which kindly lent infrastructures and material for this project. We would also like to express our gratitude to ADEMBI for their work and finally, we want to express our gratitude to BIZKAILAB initiative of the Biscay Council and the Basque Country Department of Education, Universities and Research for their support.

\section{REFERENCES}

[1] Poser, C. M., Paty, D. W., Scheinberg, L., McDonald, W. I., Davis, F. A., Ebers, G. C., ... \& Tourtellotte, W. W. 1983. New diagnostic criteria for multiple sclerosis: guidelines for research protocols. Annals of neurology, 13(3), 227-231.

[2] Amato, M. P., Ponziani, G., Rossi, F., Liedl, C. L., Stefanile, C., \& Rossi, L. 2001. Quality of life in multiple sclerosis: the impact of depression, fatigue and disability. Multiple Sclerosis, 7(5), 340-344.

[3] Ghezzi, A., Deplano, V., Faroni, J., Grasso, M. G., Liguori, M., Marrosu, G., ... \& Zaffaroni, M. 1997. Multiple sclerosis in childhood: clinical features of 149 cases. Multiple sclerosis, 3(1), 43-46.

[4] Bakshi, R. 2003. Fatigue associated with multiple sclerosis: diagnosis, impact and management. Multiple Sclerosis, 9(3), 219-227.

[5] Weinshenker, B. G., Bass, B., Rice, G. P. A., Noseworthy, J., Carriere, W., Baskerville, J., \& Ebers, G. C. 1989. The natural history of multiple sclerosis: a geographically based study I. Clinical course and disability. Brain, 112(1), 133146.

[6] Toosy, A., Ciccarelli, O., \& Thompson, A. 2013. Symptomatic treatment and management of multiple sclerosis. Handbook of clinical neurology, 122, 513-562.

[7] Lassemi, E., Sahraian, M. A., Motamedi, M. H. K., Valayi, N., \& Moradi, N. 2014. Oral and Facial Manifestations of Patients with Multiple Sclerosis. Dentistry, 4(194), 21611122.

[8] Stroud, N., Minahan, C., \& Sabapathy, S. 2009. The perceived benefits and barriers to exercise participation in persons with multiple sclerosis. Disability \& Rehabilitation, 31(26), 2216-2222.

[9] Loma, I., \& Heyman, R. 2011. Multiple sclerosis: pathogenesis and treatment. Current neuropharmacology, 9(3), 409.

[10] Rotstein, D., O'Connor, P., Lee, L., \& Murray, B. J. 2012. Multiple sclerosis fatigue is associated with reduced psychomotor vigilance. The Canadian Journal of Neurological Sciences, 39(2), 180-184.

[11] Feinstein, A. 2011. Multiple sclerosis and depression. Multiple Sclerosis Journal, 17(11), 1276-1281.

[12] Veauthier, C., Gaede, G., Radbruch, H., Gottschalk, S., Wernecke, K. D., \& Paul, F. 2013. Treatment of sleep disorders may improve fatigue in multiple sclerosis. Clinical neurology and neurosurgery, 115(9), 1826-1830.

[13] Jellie, B., Sweetland, J., Riazi, A., Cano, S.J., Playford, E.D., 2014. Staying at work and living with MS: a qualitative study of the impact of a vocational rehabilitation intervention, Disability and Rehabilitation, 1-6.

[14] Pilgrim, M. 2010. HTML5: Up and running. O'Reilly Media.

[15] A. Bangor, P. T. Kortum, J. T. Miller. 2008. An Empirical Evaluation of the System Usability Scale. International Journal of Human-Computer Interaction, 24:6, 574-594 